\title{
Research on Airport Security: A Complex Simulation Model
}

\author{
Jingxuan $\mathrm{Li}$ \\ School of Electrical and Electronic Engineering, North China Electric Power University, Beijing 102206, \\ China \\ ncepuljx@163.com
}

Keywords: Airport security, Monte Carlo simulation, Queuing theory.

\begin{abstract}
Airport security checkpoint is an important link to ensure the security during passengers' travel. However, with the increase of airports' passenger pressure, it starts to become a problem that limits passenger throughput and increases passengers' waiting time. By data analysis, it shows that ID-check time and scan time submit to normal distribution. On the basis of this, a model based on Monte Carlo simulation is established to describe security check process. By data simulation, the bottleneck of current security process is identified in zone B. More specifically, passengers spend too much time waiting in this area. Based on our previous conclusions, because the model's sensitivity of velocity is high, the first recommendation is that a separate check lane for old people should be set. In case of queue jumping phenomenon, the second recommendation is that strategies such as setting warning lines and even penalty can be taken into action. As for gentle French, because of their leisurely behaviors, the suggested arrival times for French should be ahead.
\end{abstract}

\section{Introduction}

Airport security check is extremely important to the security of airports and flights. And the time it takes related closely to passengers' arriving time before their planes take off. However, passengers' arriving time and the time they spend in checkpoint lines are their vital interests. In order to guarantee that passengers can go aboard conveniently and successfully, airport security check should increase passenger throughput, improve security check efficiency and reduce variance in wait time while maintaining security to a large extent.

\section{Data analysis}

Before establishing the model, data preprocessing is necessary. We find some data from O'Hare international airport. With the help of the information, we do the following analysis.
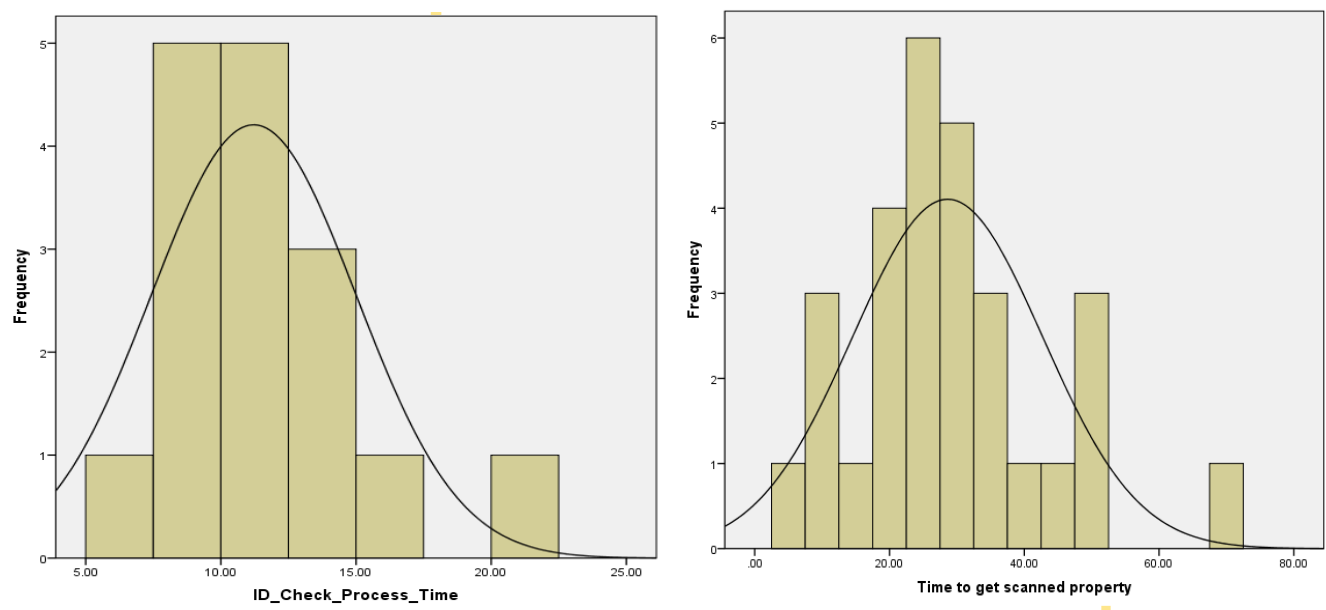

Fig. 1 Results of data analysis

We use SPSS to do normal distribution examination on data of ID check process time and time to get scanned property. The results are as follows: 
According to the validation results, we can see that two data's skewness and kurtosis indexes are all less than 1 . So it can be verified that the two data both submit to normal distribution.

\section{Model and Simulation}

We summarize the overall airport security check process in the following flow chart:

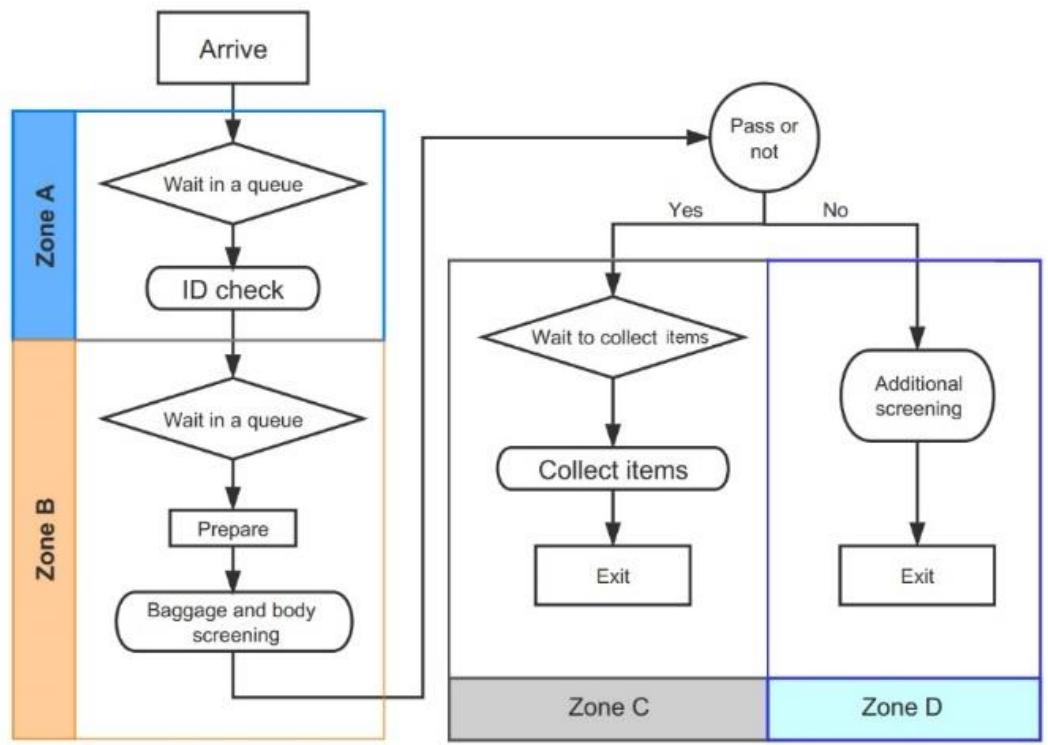

Fig. 2 Flow chart

Based on the analysis, we utilize Monte Carol algorithm to establish airport security check simulation system. This is an effective method of solving this kind of random problem.

In the model, we regard each passenger as the least unit to construct structure arrays of passengers' information. The inherent information that each passenger has is listed as follows:

Table 1 Inherent information

\begin{tabular}{|c|c|}
\hline Inherent Information & Simulation Output \\
\hline Passengers' Type & \multirow{3}{*}{ Waiting Time in Each Zone } \\
\hline Arrival Time & \\
\hline ID Check Time & \\
\hline Preparation Time(in Zone B ) & \multirow{3}{*}{ Queue Length in Each Zone } \\
\hline Scan Time & \\
\hline Collection Time(in Zone C) & \\
\hline Cutting Probability c\% & \multirow{3}{*}{ Passenger Throughput at Checkpoint } \\
\hline Forward Velocity $\mathrm{v}$ & \\
\hline Spacing d between Two Adjacent Pax & \\
\hline
\end{tabular}

Start simulation. Step length is per unit time (one second). Update the position of passengers. The simulation process of each passenger includes following steps:

Passenger start security check process according to his arrival time at zone A. Identify the type of the passenger and choose a check lane. Meanwhile, choose the shortest queue to join.

Begin ID check when the passenger arrives in the front of the queue. After the ID check is done, the passenger join the queue in zone B.

Begin preparing his belongings when the passenger arrives in the front of the queue. After preparation and scan, the passenger enters zone $C$. As for problematic passengers, they should enter zone $\mathrm{D}$ for additional checking.

After the passenger enters zone $\mathrm{C}$, whether to queue or not depends on the situation in zone $\mathrm{C}$. When the passenger can start to collect item, the total process is finished after collection. 
During the simulation process, each step length notes queuing length in each zone respectively. After finishing the simulation process of the given time period, passengers information has been collected. In addition, waiting time and the checkpoint throughput can also be obtained in this time period.

\section{Result of simulation}

We establish the distribution figure about passengers' density. The index of horizontal ordinate is time. We make it the input of our model. The distribution figure is as follows:

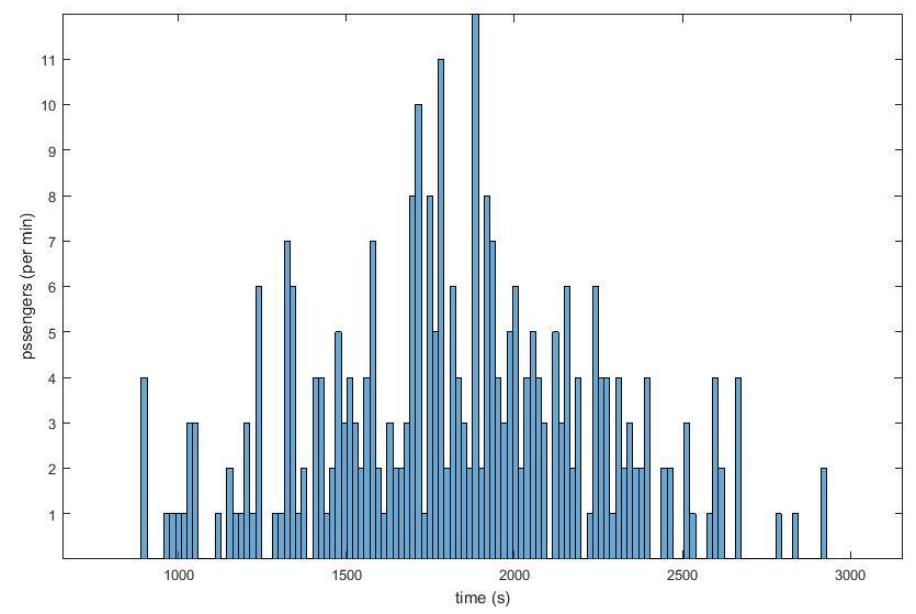

Fig. 3 Distribution
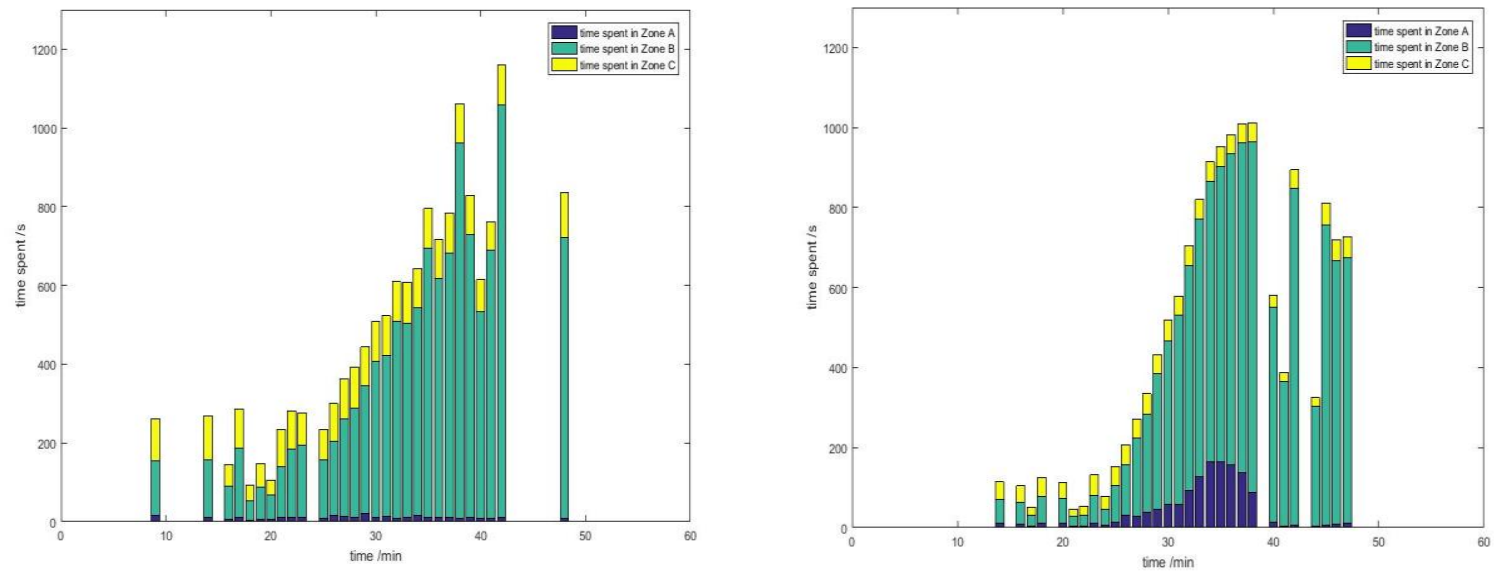

Fig. 4 Waiting times

In addition, we can obtain the regular lane's and the Pre-Check lane's waiting times in each security check zone.

\section{Summary}

In current situation, the most serious bottleneck of the current process exists in zone B. Situations in zone $\mathrm{A}$ and zone $\mathrm{C}$ have problems to some extent, too.

\section{References}

[1]. Gkitza K, Niemeier D, Mannering F. Airport security screening and changing passenger satisfaction: An exploratory assessment[J]. Journal of Air Transport Management, 2006, 12(5):213-219. 
[2]. Regattieri A 1. Designing production and service systems using queuing theory: principles and application to an airport passenger security screening system[J]. International Journal of Services \& Operations Management, 2009, (2): 206-225. 